\title{
Assessing the impact of structured education on the knowledge of hospital pharmacists about adverse drug reactions and reporting methods in Saudi Arabia: an open-label randomised controlled trial
}

\author{
Ejaz Cheema' ${ }^{1}$. Abdulmohsin Abdulaziz Almualem² Abdulmohsen Talal Basudan $^{2}$. \\ AbdulAziz Khalid Salamatullah ${ }^{2} \cdot$ Sohaib Omar Radhwi ${ }^{2} \cdot$ Ammar Soliman Alsehli $^{2}$
}

Published online: 27 March 2019

๑) Springer Nature Switzerland AG 2019

\begin{abstract}
Background Pharmacists have limited knowledge about adverse drug reactions (ADRs) in Saudi Arabia.

Objective The aim of this study was to assess the impact of educational intervention on the knowledge of hospital pharmacists about ADRs.

Methods This was a 3-month randomized controlled trial conducted in Saudi Arabia between January 2018 and March 2018. Participants in both groups were required to complete an online questionnaire at baseline and at 12-week follow-up. Participants in the intervention group received a structured information sheet about ADRs 2 weeks after the first assessment. The main outcome measure was difference in mean knowledge score about ADRs.

Main outcome measure Difference in mean knowledge score about ADRs.

Results A total of 46 participants were included in the study. At the 12-week follow-up, there was a significant improvement in the mean knowledge score ( \pm standard deviation) of intervention participants from $7.67( \pm 2.1)$ at baseline to $11.22( \pm 0.4)$ (95\% CI -4.5 to $-2.5 ; p<0.0001)$. The mean knowledge score of control participants remained unchanged at $6.71( \pm 2.3)$ during both baseline and follow-up assessments.

Conclusion ADR-specific education was associated with a significant improvement in the knowledge and understanding of pharmacists about ADRs and their methods of reporting.
\end{abstract}

Electronic supplementary material The online version of this article (https://doi.org/10.1007/s40267-019-00621-z) contains supplementary material, which is available to authorized users.

Ejaz Cheema

E.Cheema@bham.ac.uk

Abdulmohsin Abdulaziz Almualem

mr-classico@hotmail.com

Abdulmohsen Talal Basudan

abdulmohsen1996@hotmail.com

AbdulAziz Khalid Salamatullah

azizdx036@gmail.com

Sohaib Omar Radhwi

sohdawi@gmail.com

Ammar Soliman Alsehli

ammar-148@hotmail.com

1 School of pharmacy, University of Birmingham, Edgbaston, Birmingham B15 2TT, UK

2 College of pharmacy, Umm-al-Qura University, Makkah, Saudi Arabia

\section{Background}

Adverse drug reactions (ADRs) are associated with significant morbidity and mortality worldwide [1]. A study conducted to determine the number of ADR-related emergency hospital admissions in England reported an increase in ADR-led admissions from $1.2 \%$ in 2008 to $1.6 \%$ in 2015 [2]. In Saudi Arabia, the frequency of ADR-related hospital admissions was reported to be 6.1 per 100 admissions and 7.9 per 1000 patient days [3]. An ADR is defined by the Medicines and Healthcare Products Regulatory Agency (MHRA) as "an unwanted or harmful reaction experienced following the administration of a drug or a combination of drugs under normal conditions of use and which is expected to be related to the drug" [4].

Spontaneous reporting systems are the most important pharmacovigilance activity used worldwide by healthcare professionals to report any suspected ADRs that may not have been identified during premarketing clinical trials [5]. Pharmacists are also expected to play an important role in 
ensuring medicine safety by detecting and reporting ADRs. Hospital pharmacists, in particular, are ideally placed to report ADRs due to their access to patients' medical records and frequent interactions with prescribers. In the year 2009, the Saudi Food and Drug Authority (SFDA) established a National Pharmacovigilance Centre (NPC) with the aim of reporting and detecting ADRs [6]. The NPC expects all healthcare professionals, including doctors, pharmacists and nurses, to report ADR,s and have introduced both paper and online systems to facilitate ADR reporting. However, despite the availability of paper and online methods of ADR reporting, ADRs continue to be under-reported by healthcare professionals in Saudi Arabia [6].

The under-reporting of ADRs could be partly attributed to the lack of awareness and understanding of ADRs by healthcare professionals in Saudi Arabia. A cross-sectional study conducted to assess the knowledge of pharmacists about ADRs in Saudi Arabia reported inadequate knowledge and understanding about pharmacovigilance [7]. More than half of the participants of the study, including pharmacists, doctors and nurses, were not even aware of the correct definition of pharmacovigilance. Similar findings of limited awareness about pharmacovigilance and ADR reporting by healthcare professionals have been reported in another study conducted in Saudi Arabia [8]. Given the poor knowledge of healthcare professionals about ADRs and its impact on ADR reporting, this study aims to assess the impact of structured education on the knowledge of hospital pharmacists about ADRs and their reporting methods in Saudi Arabia.

\section{Methods}

This study was a 3-month randomized controlled trial conducted in the Makkah region, Saudi Arabia between January 2018 and March 2018. The study had two groups; eligible participants were subsequently randomized to either a control or an intervention group. The randomization and allocation sequence were conducted by an independent person who produced a computer-generated randomized list. This person was not involved in the recruitment or enrolment of the participants. Participants were enrolled in the study by the members of the research team. Both groups were then followed up for 3 months to see the difference in the mean knowledge score about ADRs and their methods of reporting.

\section{Study participants and procedures}

Qualified hospital pharmacists from all ethnic backgrounds working in the in-patient or out-patient settings in the private or government hospitals were eligible for the study. Eligible participants were identified and approached by the members of the research team. Exclusion criteria included community pharmacists, pharmacy students and pharmacy technicians, as well as pharmacists working in the pharmaceutical industries and academia.

A 19-item questionnaire was developed using the format and style of a questionnaire used in a previous study [9]. Specific questions were included about the methods of ADR reporting in Saudi Arabia. The questionnaire was piloted on a sample of six pharmacy students. The section "Background" had five items that explored the demographic information of participants (see Appendix 1 in the electronic supplementary material for the questionnaire). The section "Methods" comprised two main items, each having six items that aimed to gather information about the types of ADRs that should be reported by pharmacists in children and adults. The section "Results" consisted of two items that were designed to assess the awareness of pharmacists about the methods of reporting ADRs in Saudi Arabia. The maximum possible score was 14 and the minimum was 0 . The questionnaire was developed in the English language. Participants in both groups were assessed at baseline and the 12-week follow-up.

\section{Intervention and comparator}

Participants in the intervention group electronically received a double A4-sized information sheet containing structured advice on ADRs and their methods of reporting. This information was developed by a team of six researchers using the guidance produced by the SFDA and was sent to the participants two weeks after the first assessment. At the same time, a separate double A4-sized information sheet containing information about the coronavirus was also sent electronically to the control participants.

\section{Data management and analysis}

Based on the findings of a previous study [8], it was expected that $50 \%$ of the participants will be aware of the methods of ADR reporting in Saudi Arabia. Using a sample size calculator (Raosoft), the sample size calculation indicated that a sample size of 23 participants per group will provide a power of $80 \%$ at the $5 \%$ level in a 2-tailed test to detect an increase in the participants' awareness about methods of ADR reporting from 50 to $90 \%$. Questionnaire responses were coded, and data was analysed using SPSS version 22. Data was single-entered. Paired $T$ test was used to compare the mean knowledge score of the participants within the group (at baseline and followup) and un-paired $T$ test was used to compare the score between the two groups. 


\section{Results}

A total of 55 participants were invited to take part in the study. Of these, 46 participants agreed to take part and were included in the study (response rate $84 \%$ ); see Fig. 1 for the flow of participants through the study. At baseline, no statistically significant differences were found between the demographics of participants in the intervention and the control groups (Table 1).

\section{Impact on types of adverse drug reactions (ADRs) likely to be reported by pharmacists and methods of reporting}

At the 12-week follow-up, there was a significant improvement in the percentage of participants who were familiar with the types of ADRs that should be reported in adults (section 2 of the questionnaire in supplementary material) in the intervention group (from 0 at baseline to $28 \%$; $95 \%$ CI $6.6-51.0 ; p=0.008)$. There was a non-significant improvement in the percentage of control participants from $4 \%$ at baseline to $12.5 \%$ at the follow-up for the same questionnaire item (95\% CI -9.8 to $32.2 ; p=0.032)$. Similarly, there was a significant improvement in the percentage of intervention participants who were familiar with the types of ADRs that should be reported in children from $4.5 \%$ at baseline to $28 \%$ at follow-up (95\% CI 0.4-46.6; $p=0.04$ ). However, the percentage of control participants did not improve significantly ( $0 \%$ at baseline to $6 \%$ at the follow-up) $(95 \%$ CI -8.6 to $28.0 ; p=0.23$ ) for the same questionnaire item.

With regards to the awareness about methods of ADR reporting (section 3 of the questionnaire in supplementary material), a significant improvement was reported in the awareness of intervention participants from $13.6 \%$ at baseline to $61.1 \%$ at the follow-up (95\% CI 18.0-68.4; $p=0.0018$ ) as comparted with control participants who only reported a non-significant improvement from $20.8 \%$ at baseline to $25 \%$ at follow-up (95\% CI -20.3 to $31.2 ; p=0.75$ ).

\section{Impact on the mean knowledge score about ADRs}

At the 12-week follow-up, there was a significant improvement in the mean knowledge score ( \pm standard deviation) of intervention participants from $7.67 \pm 2.1$ at baseline to $11.22 \pm 0.4(95 \% \mathrm{CI}-4.5$ to $-2.5 ; p<0.0001)$. The mean knowledge score of control participants remained unchanged at $6.71 \pm 2.3$ during both baseline and follow-up assessments.

\section{Discussion}

To the authors' knowledge, this is the first RCT that has assessed the impact of ADR-specific education on the knowledge of hospital pharmacists about ADRs and the methods of reporting them in Saudi Arabia. This study reported that

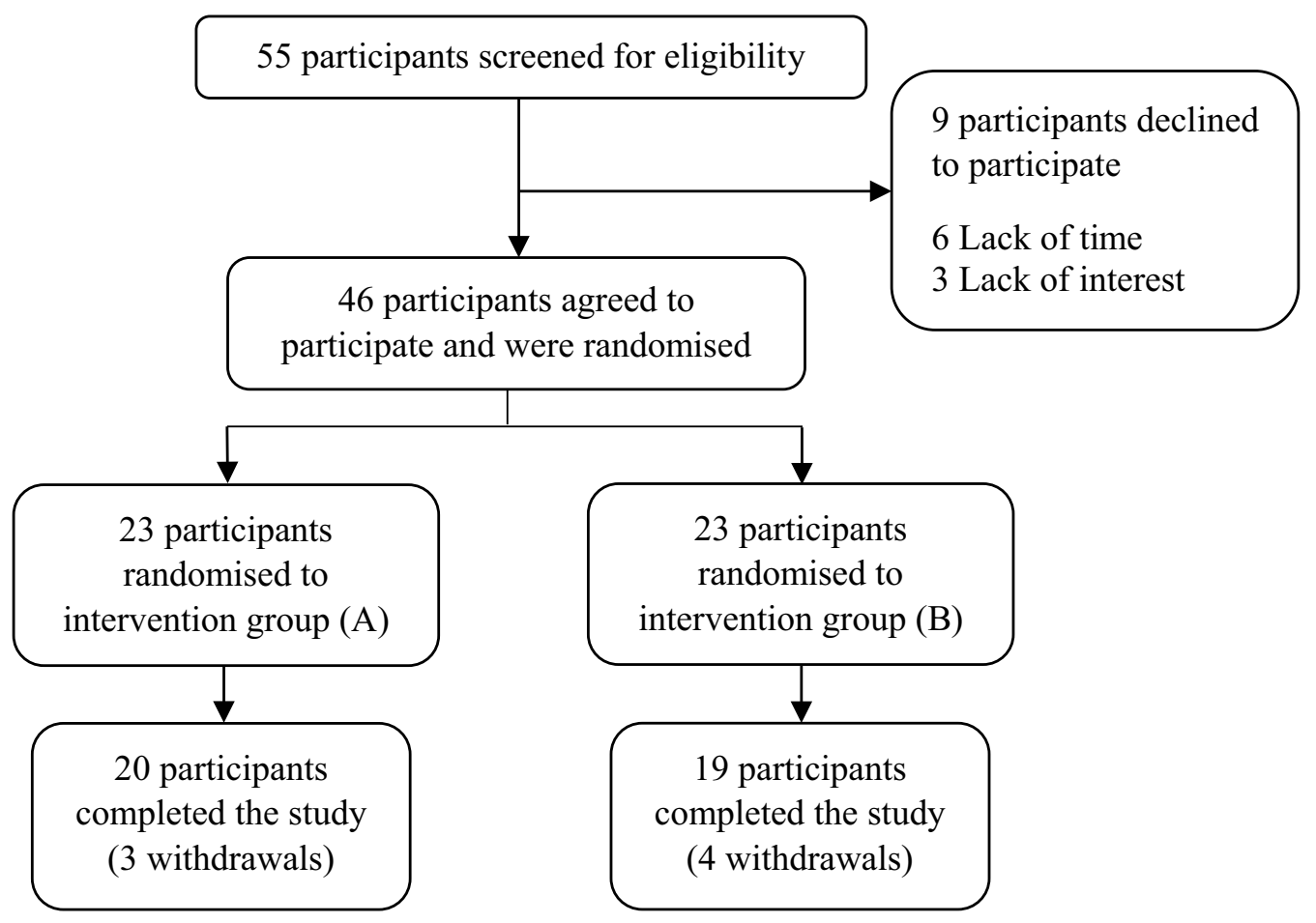

Fig. 1 Flow of participants through the study 


\section{Table 1 Participant demographics at baseline}

\begin{tabular}{|c|c|c|c|}
\hline \multirow[t]{2}{*}{ Variable } & \multicolumn{2}{|c|}{ No. of participants $(\%)^{\mathrm{a}}$} & \multirow[t]{2}{*}{$\mathrm{BGD}(p$ value $)$} \\
\hline & $\begin{array}{l}\text { Intervention } \\
(n=23)\end{array}$ & $\begin{array}{l}\text { Control } \\
(n=23)\end{array}$ & \\
\hline \multicolumn{4}{|l|}{ Age (years) } \\
\hline $20-23$ & 0 & $1(4)$ & 0.33 \\
\hline $24-27$ & $10(43)$ & $8(35)$ & 0.58 \\
\hline $28-31$ & $8(35)$ & $11(48)$ & 0.37 \\
\hline$\geq 32$ & $5(22)$ & $3(13)$ & 0.42 \\
\hline \multicolumn{4}{|l|}{ Gender } \\
\hline Male & $12(52)$ & $13(57)$ & 0.73 \\
\hline Female & $11(48)$ & $10(43)$ & 0.73 \\
\hline \multicolumn{4}{|c|}{ Type of organization } \\
\hline Public & $23(100)$ & $23(100)$ & \\
\hline Private & 0 & 0 & \\
\hline \multicolumn{4}{|c|}{ Years since qualification } \\
\hline$<1$ & 0 & $3(13)$ & 0.07 \\
\hline $1-5$ & $14(61)$ & $8(35)$ & 0.08 \\
\hline $6-10$ & $8(35)$ & $9(39)$ & 0.78 \\
\hline$\geq 11$ & $1(4)$ & $3(13)$ & 0.27 \\
\hline \multicolumn{4}{|l|}{ Knowledge score } \\
\hline Mean score \pm SD & $7.67 \pm 2.1$ & $6.71 \pm 2.3$ & 0.66 \\
\hline
\end{tabular}

$B G D$ between-group difference, $S D$ standard deviation

${ }^{\mathrm{a}}$ Unless otherwise indicated

provision of ADR-specific education was associated with a significant improvement in the mean knowledge score of intervention participants compared with the participants in the control group. Furthermore, the educational intervention also led to a significant increase in the awareness of pharmacists about methods of ADR reporting. Similar findings have also been previously reported in a cluster RCT that reported a significant improvement in ADR reporting by pharmacists who received an educational programme about pharmacovigilance and ADRs [10].

Provision of structured and written education on ADRs to participants in the intervention group was associated with a significant improvement in their knowledge about the types of ADRs that should be reported in adults (from 0 to 28\%) and children (from 4.5 to $28 \%$ ) as opposed to control participants who only showed a non-significant improvement in their knowledge. Although considerable improvement was reported in the knowledge of intervention participants, a majority of the participants (around 70\%) failed to correctly identify the types of ADRs that should be reported in adults and children. A majority of the participants did not consider it important to report mild reactions from a drug with a black triangle in adults during both pre- and postintervention assessments. As far as reporting of ADRs in children was considered, reporting of mild reactions from an existing drug was not considered to be important by most of the pharmacists who completed the study. One of the possible reasons that may explain such a misconception by intervention participants about ADR reporting is that perhaps they needed further reminders about ADRs and the types of ADRs that should be reported in both adults and children. It is important to highlight that participants in the intervention group only received the educational intervention once at the start of the study and did not receive any further education during the rest of the study period. With regards to the awareness of participants about the methods of ADR reporting, a majority $(\sim 90 \%)$ were only aware about the online method of reporting prior to receiving educational intervention. Following the provision of ADR-specific education, more than half $(61 \%)$ of the participants were reported to be aware of both paper-based and online methods of ADR reporting. Awareness of control participants about ADR reporting methods remained largely unchanged between baseline and follow-up assessments of the study.

The effectiveness of the educational intervention in improving the mean knowledge score of pharmacists about ADRs underscores the importance of providing explicit education to pharmacists about ADRs at both undergraduate and practice level. Topics related to pharmacovigilance are not given due share in the curricula offered by the majority of institutions offering medicine, pharmacy or nursing programmes in Saudi Arabia [7]. This could be explained by the lack of availability of enough qualified staff trained in pharmacovigilance and medication safety in Saudi Arabia [6]. The NPC, therefore, needs to make efforts to introduce the concept of pharmacovigilance to healthcare professionals working in the hospital settings by organizing specific educational seminars and workshops. Hospital pharmacists by virtue of their regular contact with patients together with access to medical records are ideally placed to report suspected ADRs and should therefore be encouraged to improve their ADR reporting. Provision of continuous professional development programmes to pharmacists can help address their knowledge gaps in ADR detection and further improve ADR reporting. The aim of such programmes should not only be to improve pharmacists' understanding about ADRs, but should also focus on changing their attitudes and perceptions toward ADR reporting. Furthermore, core topics related to pharmacovigilance should be included in the curricula offered by the academic institutions to enhance the knowledge of healthcare students about ADR reporting.

This study was limited by using non-validated information sheets that were delivered to study participants. Furthermore, participants could not be blinded to the study intervention owing to the nature of educational interventions. Nevertheless, this study has several strengths. It was a well-designed RCT that was informed by prior evidence. A sample size calculation was undertaken prior to the study. Exclusion and inclusion criteria were rigorously applied to 
ensure that the study population was representative of the target population. Participants were randomly allocated to the study arms through a set of computer-generated numbers to minimize selection bias.

\section{Conclusion}

The findings of this study suggest that ADR-specific education can improve the knowledge and understanding of pharmacists about ADRs and their methods of reporting. Future work should focus on the development of effective instruction methods that deliver pharmacovigilance education to healthcare professionals with the aim of improving their ADR reporting in Saudi Arabia.

Acknowledgements We would like to thank all individuals who participated in the study.

\section{Compliance with ethical standards}

Ethics approval The study was approved by the Institutional Review Board of Umm-al-Qura University (UQU-COP-EA\# 143630).

Conflict of interest The authors declare no relevant conflicts of interest

Funding This study did not receive any funding.

Informed consent Participants were provided written information about the study and its aims. The completion and submission of the anonymised online questionnaire by the participants was taken as their consent to the study.

\section{References}

1. Davies EC, Green CF, Taylor S, et al. Adverse drug reactions in hospital inpatients: a prospective analysis of 3695 patient-episodes. PLoS One. 2009;4:e4439.

2. Vereen JC, Weiss M. Trends in emergency hospital admissions in England due to adverse drug reactions: 2008-2015. JPHS. 2017;8:5-11.

3. Aljadhey H, Mahmoud MA, Ahmed Y, et al. Incidence of adverse drug events in public and private hospitals in Riyadh, Saudi Arabia: the (ADESA) prospective cohort study. BMJ Open. 2016;6:e010831. https://doi.org/10.1136/bmjopen-2015-010831.

4. MHRA. Adverse Drug Reactions. http://www.mhra.gov.uk/Safetyinformation/Howwemonitorthesafetyofproducts/Medicines/ TheYellowCardScheme/Informationforhealthcareprofessionals/ Adversedrugreactions/index.htm. Accessed 01 October 2018.

5. Hazell L, Shakir SA. Under-reporting of adverse drug reactions: a systematic review. Drug Saf. 2006;29:385-96.

6. Alshammari TM, Alshakka M, Aljadhey H. Pharmacovigilance system in Saudi Arabia. Saudi Pharm J. 2017;25:299-305. https ://doi.org/10.1016/j.jsps.2016.09.008.

7. Al Doughan FF, Alomi YA, Iflaifel MH. Pharmacist's awareness and knowledge of reporting adverse drug reactions in Saudi Arabia. Int J Pharm Health Sci. 2019;2:60-5.

8. Alharbi F, Bahnassi A, Alonazie W. Attitude, knowledge and experience of hospital pharmacists with pharmacovigilance in a region in Saudi Arabia: a cross-sectional study. Trop J Pharm Res. 2016;15:1773-9.

9. Cheema E, Haseeb A, Khan TM, et al. Barriers to reporting of adverse drugs reactions: a cross sectional study among community pharmacists in United Kingdom. Pharm Pract. 2017;15:931.

10. Herdeiro MT, Polonia J, Gestal-Otero JJ, et al. Improving the reporting of adverse drug reactions: a cluster-randomized trial among pharmacists in Portugal. Drug Saf. 2008;31:335-44. 